\title{
Protons and more: state of the art in radiotherapy
}

\author{
Authors: Peter ] Hoskin ${ }^{A}$ and Indrani S Bhattacharya ${ }^{B}$
}

The purpose of modern radiotherapy is to deliver a precise high dose of radiation which will result in reproductive death of the cells. Radiation should transverse within the tumour volume whilst minimising damage to surrounding normal tissue. Overall $40 \%$ of cancers which are cured will have received radiotherapy. Current state of the art treatment will incorporate cross-sectional imaging and multiple high energy $X$-ray beams in processes called intensity modulated radiotherapy and image guided radiotherapy. Brachytherapy enables very high radiation doses to be delivered by the direct passage of a radiation source through or within the tumour volume and similar results can be achieved using rotational stereotactic X-ray beam techniques. Protons have the characteristics of particle beams which deposit their energy in a finite fixed peak at depth in tissue with no dose beyond this point the Bragg peak. This has advantages in certain sites such as the spine adjacent to the spinal cord and particularly in children when the overall volume of tissue receiving radiation can be minimised.

KEYWORDS: Intensity modulated radiotherapy, image guided radiotherapy, brachytherapy, stereotactic radiotherapy, protons

\section{Introduction}

Treatments for cancer can be subdivided into local, locoregional and systemic. Radiotherapy and surgery provide locoregional and local control, while systemic therapy is achieved with chemotherapy, hormonal therapy and biological agents.

Radiotherapy has been utilised as a treatment for cancer for over 100 years, dating back to the discovery of X-rays in $1895{ }^{1}$ It was Marie Curie who discovered the radioactive elements polonium and radium, following which radiation therapy began. Radiotherapy remains the most effective non-surgical treatment in the management of cancer, with approximately $60 \%$ of cancer patients receiving radiation therapy during their illness and $75 \%$ of these patients being treated with curative intent. ${ }^{2}$ Radiotherapy is essential for the effective palliation of pain, haemorrhage, obstructive symptoms and neurological complications. Cancer will remain a major burden in the years ahead with the worldwide incidence trending upwards. While access to modern radiotherapy is therefore an essential

Authors: ${ }^{\text {A }}$ consultant clinical oncologist, Mount Vernon Cancer Centre, Middlesex, UK; ${ }^{B}$ specialist registrar in clinical oncology, Mount Vernon Cancer Centre, Middlesex, UK component of any healthcare system, there are many challenges in delivering high quality modern day radiotherapy. These include developing techniques to conform high dose envelopes around irregular tumour volumes, creating reliable and reproducible multifraction schedules, and optimising the use of expensive equipment. As we develop new techniques, including intensity modulated radiotherapy (IMRT), image guided radiotherapy (IGRT), stereotactic radiotherapy and proton therapy, we strive to provide state of the art radiotherapy for all.

\section{Radiation principles}

The principle of radiotherapy is to deliver ionising radiation to a tumour that will result in reproductive death of the cell. In doing so, the radiation dose to immediate normal surrounding tissues (ie organs at risk) should be kept to a minimum. X-ray beams will be generated at various energies. Low energy beams (up to $300 \mathrm{kV}$ ) will be useful only for superficial structures. Most radiotherapy today, aiming at internal organs, will use megavoltage X-rays of 4-20 million volts produced by a linear accelerator (Fig 1). X-rays undergo a continuous process of attenuation as they move away from their generating source, and thus a high initial intensity is required which continues to deliver ionising radiation through and beyond the treated site. This is the 'exit beam'. Alternatively, ionising radiation can also be delivered by particle beams that deposit their energy in a much more focused way to a fixed depth beyond which there is

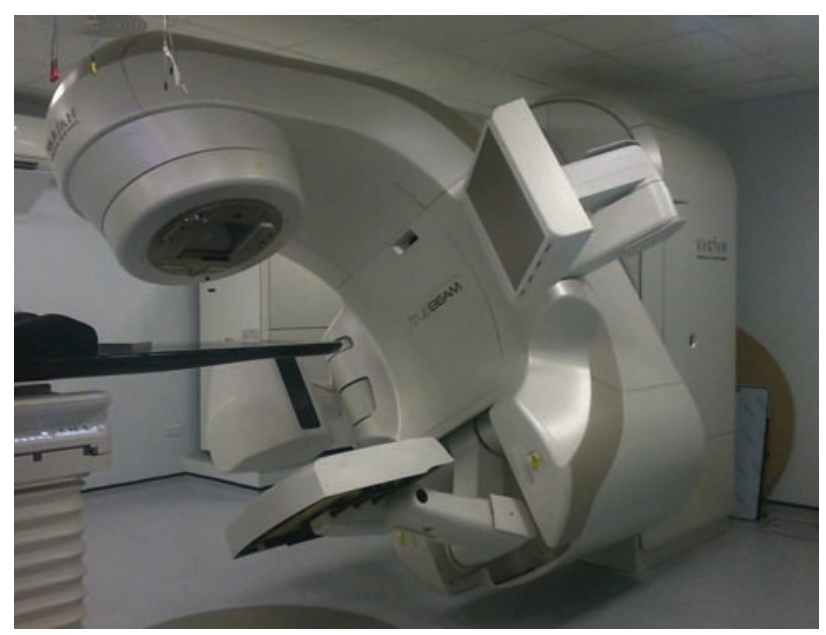

Fig 1. Linear accelerator at Mount Vernon Cancer Centre. 
Fig 2. The Bragg peak demonstrating the energy loss of ionising radiation during its travel through matter with the peak or maximum energy deposition occurring immediately before the particle comes to rest.

Reproduced with permission from www.floridaproton.org/what-isproton-therapy.

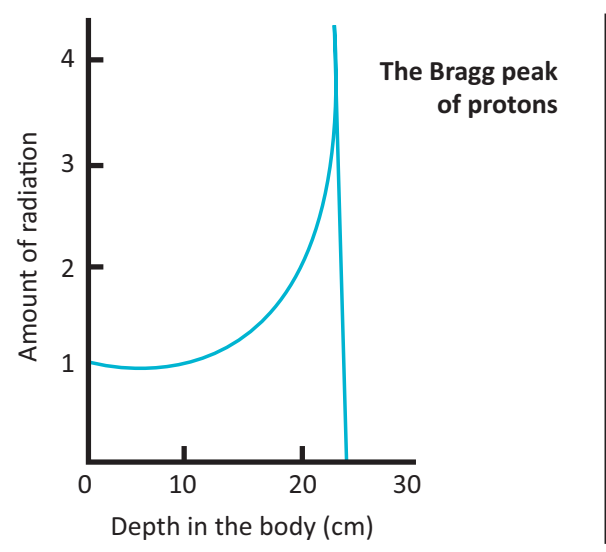

little or no radiation exposure. The common particle beam used in clinical practice is an electron beam, which will penetrate a few centimetres only and is therefore ideal for skin tumours and superficial structures. Protons are a type of particle beam which also deliver radiation to a fixed depth known as the Bragg peak. The advantage of this type of beam is that although there is radiation during the entry phase to the body, there is no significant dose delivered beyond the Bragg peak. The depth at which energy is deposited with such beams is governed by the accelerating energy; very high energy proton beams of over 100 million volts are required to reach internal organs (Fig 2).

\section{External beam radiotherapy}

External beam radiotherapy (EBRT) is the mainstay of radiation therapy, comprising $85 \%$ of all therapeutic radiation exposures. Delivering a uniform, accurate dose of radiation using EBRT is a complex process requiring a skilled team of physicians, physicists and radiographers. While for simple palliative treatments single beams or two 'opposed' beams (ie coming from front and back to meet in the middle) are used, most radiotherapy now harnesses sophisticated computerised algorithms and millimetre precision to achieve optimal delivery while minimising normal tissue doses.

Three-dimensional (3D) conformal radiotherapy incorporates axial imaging data, allowing $3 \mathrm{D}$ reconstruction of the tumour and surrounding organs. This ensures accurate localisation of the target volume and identifies the amount of normal tissue that will be irradiated. The number and profile of the radiation beams can be orientated and shaped to fit the profile of the target from a beam's eye view using a multi-leaf collimator ${ }^{1}$

(Fig 3). The resulting radiotherapy plan is displayed as a colour map of radiation doses overlaid onto the anatomical computed tomography (CT) images (Fig 4). This determines whether the tumour will receive sufficient irradiation and whether acceptable doses within tolerance will have been received by normal tissues. The object is to deliver high tumour radiation doses while minimising doses to the sensitive surrounding normal tissues.

\section{Intensity modulated radiotherapy}

Intensity modulated radiotherapy (IMRT) uses multiple radiation beams which are not only shaped and orientated to conform to the tumour volume, but also modulated with respect to the intensity of radiation across each treatment beam. IMRT produces dose distributions that conform to highly complex shapes. This allows the treatment of tumour volumes that wrap around sensitive normal structures, such as the spinal cord, and thus high doses can be delivered to the tumour volume while sparing doses to the normal structures. IMRT typically uses between five and nine beams focused on the target. ${ }^{3-5}$

Although IMRT has improved conformation to irregular tumour volumes, the use of multiple beams with both entry and exit dose delivery results in an extensive low dose area around the tumour (Fig 5). The long term significance of this low dose bath effect is uncertain, but there are concerns that it could theoretically increase the risk of induced secondary malignancy. Currently, follow-up from clinical studies is too short and patient numbers too small to really determine whether there is a risk from our more modern irradiation techniques with X-rays, which translate into clinically significant increases in the development of a second cancer. ${ }^{6}$

\section{Image guided radiotherapy}

In external beam radiotherapy, the treatment volume and subsequent calculations defining the beam characteristics and

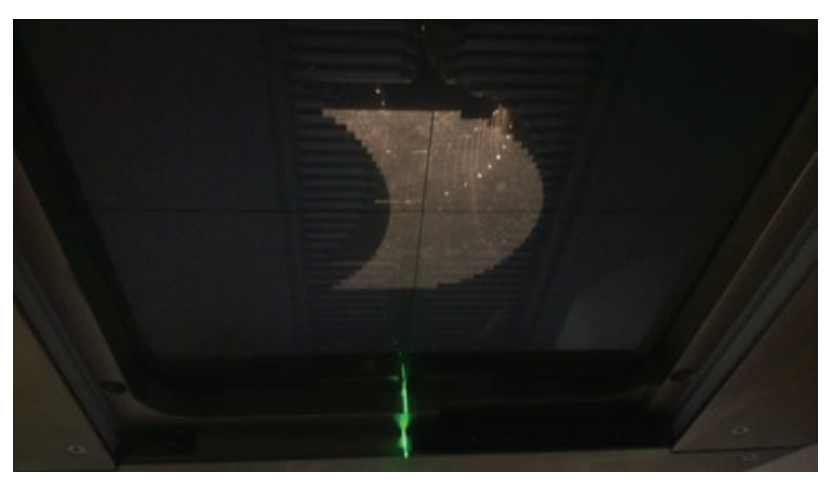

Fig 3. MLC. The MLC is made up of individual 'leaves' which can move independently in and out of the path of a radiation beam in order to block it. This device is situated in the head of a linear accelerator to shape the treatment beam in order to match the borders of the target tumour. $\mathrm{MLC}=$ multi-leaf collimator. 


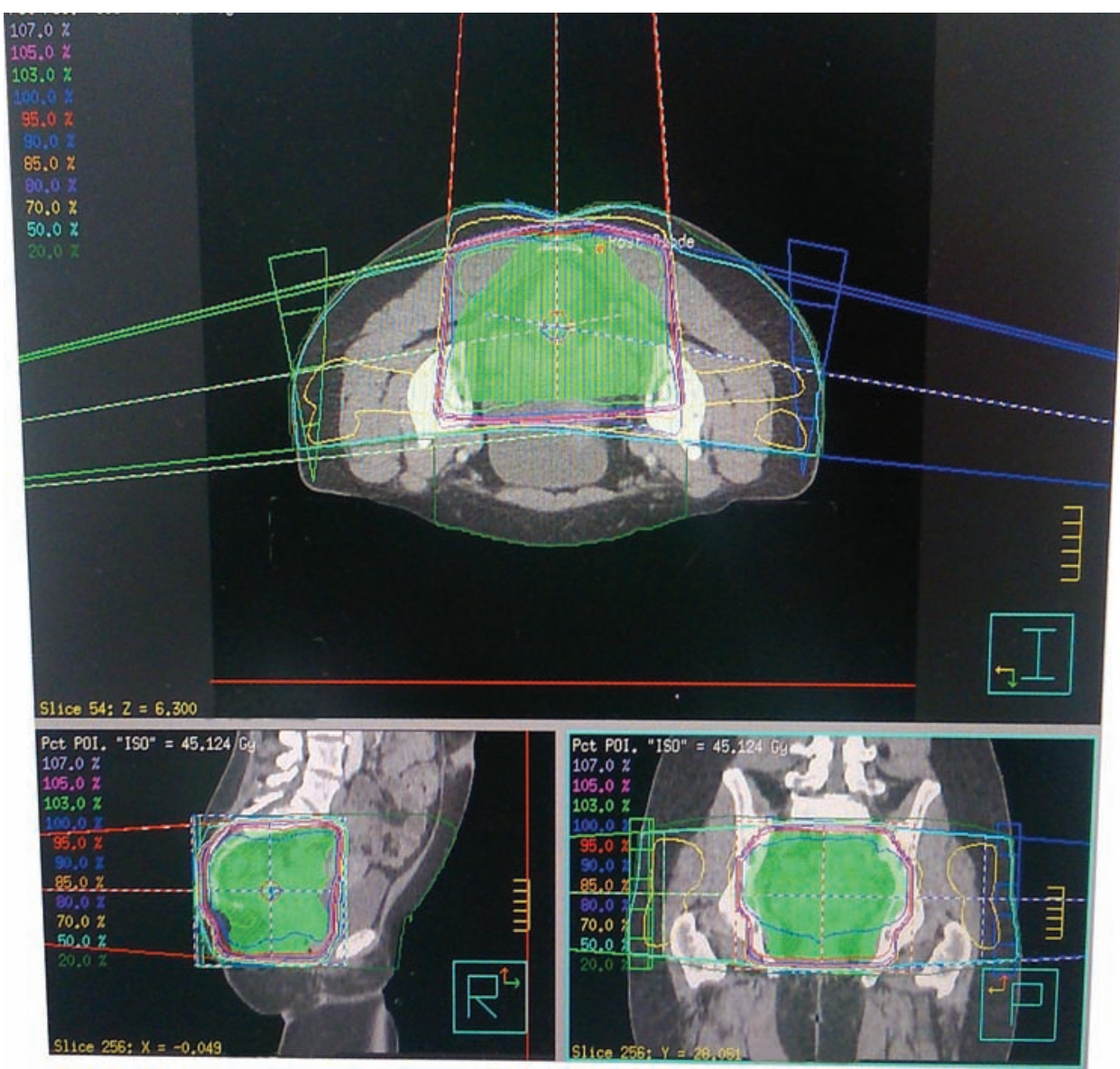

Fig 4. A three-dimensional conformal radiotherapy plan for treatment of a rectal cancer. The planning target volume is defined by the shaded green region. Three treatment beams overlap over the target volume to provide the desired dose in this region with reduced dose to surrounding normal structures. The coloured lines represent the dose gradient in a similar way to the contour lines on a map and correspond to the numbers in the top left corner. positions are based on CT images. The CT used is a snapshot of the position of the patient and anatomy at a single point in time. Internal organs are subject to a degree of movement, which can occur day-to-day and hour-to-hour. Inter-fraction motion refers to degrees of movement on a daily basis (ie between fractions), and intra-fraction motion refers to degrees

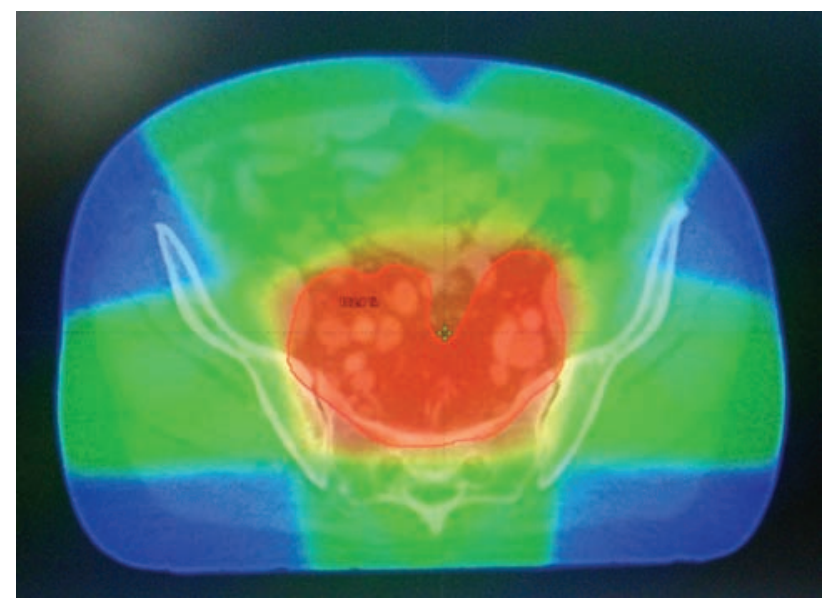

Fig 5. IMRT plan of prostate gland showing multiple beams to achieve precise dose painting across the planning target volume with a lowdose bath effect. IMRT = intensity modulated radiotherapy. of movement which occur during treatment delivery. Some organs such as the lung may have predictable movements, whereas organs such as the bowel can have random movements occurring due to the passage of gas and faeces. Larger margins are added to the target volume to account for these movements, however the addition of margins to the tumour target volume inevitably increases the volume of normal tissue treated and may increase the dose to organs at risk.

Image guidance is the process of locating the exact 3D position of the tumour and surrounding organs during treatment. This information is then used to correct for any deviation from the original plan. Fiducial markers can be implanted in certain tumour sites (eg prostate) in order to localise the treatment. ${ }^{7}$ This optimises the chance of accurately targeting the tumour and minimises the dose to the surrounding normal tissue.

IGRT entails many different imaging strategies which include planar (2D) imaging, volumetric (3D) imaging or imaging over time (4D) during the radiotherapy. Modern linear accelerators have the capacity to acquire X-rays and CT images of the area being treated, which are used to verify accurate delivery.

\section{Stereotactic radiotherapy}

Stereotactic radiotherapy may be achieved using a number of different approaches. These may involve modification of a standard linear accelerator delivery as in RapidArc, VMAT and 
tomotherapy, or specialised machines such as Gammknife or Cyberknife. Stereotactic body radiotherapy (SBRT) is based on stereotactic radiosurgery methods for the treatment of intracranial tumours. SBRT is only appropriate for selected, small extracranial lesions such as tumours of the lung and prostate or small solitary metastases. Gammaknife is an option for benign or malignant brain tumours up to $4 \mathrm{~cm}$ in size, and vascular malformations. Gammaknife contains 201 cobalt- 60 sources each placed in a circular array in a heavily shielded assembly. This delivers radiation to a target point in the patient's brain. A metal frame is surgically fixed to the patient's skull to ensure the lesion remains stationary at the target point of the radiation. Cyberknife is a frameless robotic surgery system used for treating lesions both in the brain and within the body. It uses precise information on tumour position and delivers radiation using multiple beams, but has the disadvantage of long treatment times which may be up to 1 hour (Fig 6).

The precision of SBRT allows high doses to be delivered in a very limited number of fractions, where doses per fraction can be as high as $20 \mathrm{~Gy}$. The mechanism of tumour cell kill is thought to be different at such high doses. It is speculated that radiation doses of more than 10 Gy per fraction can cause rapid obliteration of tumour vasculature, which cannot be achieved with lower conventional doses.

\section{Brachytherapy}

Brachytherapy uses a radioisotope placed on the surface of or inside the patient. The radiation source is sealed and emits ionising radiation in the form of $\gamma$ radiation to the surrounding tissues. Interstitial brachytherapy is achieved by placing the source into the target tissues or tumour itself, for example in breast and prostate. Intracavitary and intraluminal brachytherapy is where the source is placed into a body cavity such as the uterine cavity, oesophagus or bronchus. One of the key advantages of brachytherapy over external beam techniques is that the radiation only affects a very localised area around the source as dose falls off rapidly, according to the inverse square law. This allows very high doses to be administered to the target volume.

Brachytherapy can be classified by the rate at which the radiation dose is delivered. Low dose rate (LDR) brachytherapy sources emit radiation at a rate of up to $2 \mathrm{~Gy} / \mathrm{h}$. LDR sources can be permanent, such as iodine-125 seeds with prostate

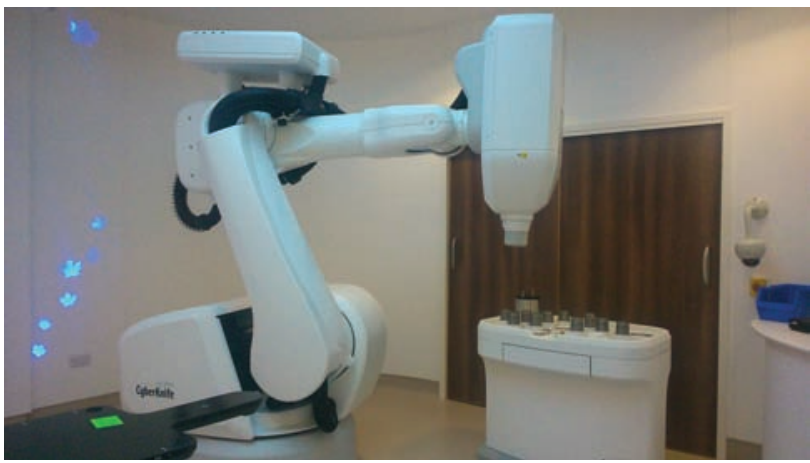

Fig 6. Cyberknife at Mount Vernon Cancer Centre. brachytherapy. High dose rate (HDR) brachytherapy sources emit radiation at a rate of over $12 \mathrm{~Gy} / \mathrm{h}$. HDR sources are always 'afterloaded'. This is where hollow catheters are inserted which will carry the source to the target volume when subsequently connected to the HDR unit, which is operated remotely to deliver the source into each catheter. Until the time of exposure, when the source is passed through the catheter in the HDR unit for a few minutes, there is no radiation exposure. The HDR afterloader holds the source within a shielded safe and passes it along the catheters to a defined position and for a calculated duration to deliver the planned radiation dose. The operator is outside the shielded room. Iridium-192 sources are most commonly used in HDR brachytherapy in gynaecological, breast and prostate brachytherapy.

\section{Particle therapy}

Particle therapy is also known as hadron therapy. This is where highly energetic particles such as electrons, protons, neutrons or positive ions are directed at the tumour. Electrons tend to be considered separately and are used for skin tumours and superficial tumours as they penetrate a few centimetres only. Another form of particle therapy uses protons which are accelerated by a cyclotron or synchrotron. Protons have certain advantages over photons. Protons penetrate deep into body tissues depositing the majority of energy in the last few millimetres of their range. ${ }^{8}$ There is almost no radiation passing beyond this distance. Therefore, by varying the proton beam energy the depth of treatment can be controlled. In most treatments, protons of different energies are used to treat the whole tumour. Protons have a greater mass and therefore scatter less with a more focused beam. This is important where it is essential to limit the radiation dose to a critical normal structure which lies deep to the tumour. An example of this is where the spinal cord lies immediately behind the target. The minimal exit dose from proton therapy can prevent long term morbidity. This is particularly important for paediatric neoplasms such as medulloblastomas where there is convincing data demonstrating the advantage of sparing the developing brain and spinal cord. ${ }^{10-12}$ The rate of secondary malignancy is theoretically less with proton therapy than IMRT as the overall dose to surrounding tissues is reduced. ${ }^{13}$

Low energy protons can be used in choroidal melanomas with good local control and preservation of the eye and sight. ${ }^{14}$ Low energy protons are available in the UK at Clatterbridge Centre for Oncology but there are no high energy proton facilities currently in this country. In April 2008, the National Specialised Commissioning Team established a programme to send patients overseas for proton beam therapy. A clinical panel reviews individual cases and if proton therapy is deemed to be a suitable treatment, an overseas referral is made which is funded by the NHS. Currently NHS patients are referred to either the University of Florida Proton Therapy Institute in Jacksonville or the ProCure Proton Therapy Centre in Oklahoma City and around 400 patients per year are approved to receive this treatment.

In line with the government policy Improving outcomes: A strategy for cancer (2011), the Department of Health plans to develop a service treating up to 1,500 patients a year over two sites - The Christie, Manchester and University College London Hospitals NHS Foundation Trust. These services will 
be available in 2018. Proton therapy incurs significant costs. The cost of a cyclotron is approximately US $\$ 180$ million, which contrasts dramatically with the cost of a state of the art linear accelerator for photon treatment at approximately $\mathfrak{E} 1.5$ million.

Only a few centres in the world have the capability to treat patients with other forms of particle therapy. Fast neutron therapy and carbon ion therapy are in clinical use. These have physical advantages similar to protons where there is sparing of tissues at depth, but there are additional biological benefits. Neutrons and carbon ions cause dense ionisation with much greater transfer of energy along the radiation track resulting in more radiation damage and cell death. ${ }^{15}$ There is also theoretical evidence that intensely ionising radiation can overcome the detrimental effects of tumour hypoxia, which is a major cause of treatment failure with standard radiotherapy. ${ }^{16}$

\section{Conclusion}

As the burden of cancer rises, there is an increasing challenge to provide state-of-the-art radiotherapy. A close relationship between diagnostic and therapeutic radiation technologies is essential. ${ }^{18}$ In recent times, the majority of advances in radiotherapy have been in response to improved imaging which enables accurate 3D image acquisition. Modern radiotherapy demonstrates the application of state-of-the-art imaging for diagnosis, target and organ at risk volume definition, tissue density measurements and verification of the therapeutic beam delivery. Radiotherapy is a highly cost-effective modality of cancer treatment. There is increasing evidence to show that IGRT, IMRT and stereotactic radiotherapy are superior methods of treatment and these techniques can achieve cure in $40 \%$ of cancer patients in contrast to chemotherapy which is responsible for $<10 \%$ of cures, despite a much greater public profile and resource investment. Proton beam therapy will be available in the UK within the next 4 years, complementing the high quality techniques already available, such as IMRT with IGRT, brachytherapy and SBRT. Further advances in the sophistication of our radiotherapy techniques will enable us to continue to provide state of the art radiotherapy and deliver a high quality service for all.

\section{References}

1 Alonzi R, Hoskin P. Imaging for radiotherapy. In: Adam A, Dixon A, Gillard J, Schaefer-Prokop C (eds), Grainger and Allison's Diagnostic Radiology, 6th edn. Oxford: Elsevier, 2014: Chapter 73, in press.

2 American Medical Association. Physician Characteristics and Distribution in the US, 2012. Chicago: AMA, 2012.
3 Staffurth J. A review of the clinical evidence for intensity-modulated radiotherapy. Clin Oncol (R Coll Radiol) 2010;22:643-57.

4 Jin JY, Wen N, Ren L et al. Advances in treatment techniques: arc-based and other intensity modulated therapies. Cancer J 2011;17:166-76.

5 Kupelian P, Langen K. Helical tomotherapy: image-guided and adaptive radiotherapy. Front Radiat Ther Oncol 2011;43:165-80.

6 Murray L, Henry A, Hoskin P et al. Second primary cancers after radiation for prostate cancer: a review of data from planning studies. Radiat Oncol 2013;8:172.

7 National Radiotherapy Implementation Group. Image guided radiotherapy (IGRT), guidance for implementation and use. London: National Cancer Action Team, 2012.

8 Tipton K, Launders JH, Inamdar R et al. Stereotactic body radiation therapy: scope of the literature. Ann Intern Med 2011; 154:737-45.

9 Terasawa T, Dvorak T, Ip S et al. Systematic review: charged-particle radiation therapy for cancer. Ann Intern Med 2009;151:556-65.

10 Blomstrand M, Brodin NP, Munck Af Rosenschöld P et al. Estimated clinical benefit of protecting neurogenesis in the developing brain during radiation therapy for pediatric medulloblastoma. Neuro Oncol 2012;14:882-9.

11 Brodin NP, Vogelius IR, Maraldo MV et al. Life years lost comparing potentially fatal late complications after radiotherapy for pediatric medulloblastoma on a common scale. Cancer 2012;118:5432-40.

12 Kuhlthau KA, Pulsifer MB, Yeap BY et al. Prospective study of health-related quality of life for children with brain tumors treated with proton radiotherapy. J Clin Oncol 2012;30:2079-86.

13 Fontenot JD, Lee AK, Newhauser WD. Risk of secondary malignant neoplasms from proton therapy and intensity modulated $\mathrm{x}$-ray therapy for early state prostate cancer. Int J Radiation Oncology Biol Phys 2009;74:616-22.

14 Damato B, Kacperek A, Chopra M et al. Proton beam radiotherapy of choroidal melanoma: The Liverpool-Clatterbridge experience. Int J Radiat Oncol Biol Phys 2005;62:1405-11.

15 Ando K, Kase Y. Biological characteristics of carbon-ion therapy. Int J Radiat Biol 2009;85:715-28.

16 Wenzl T, Wilkens JJ. Modelling of the oxygen enhancement ratio for ion beam radiation therapy. Phys Med Biol 2011;56:3251-68.

17 Wilkins A, Parker C. Treating prostate cancer with radiotherapy. Nat Rev Clin Oncol 2010;7:583-89.

18 Kenny LM, Adam A. Radiation oncology and interventional oncology: time for a collaborative approach. Clin Oncol 2013;25:515-8.

Address for correspondence: Prof PJ Hoskin, Mount Vernon Cancer Centre, Rickmansworth Road, Northwood, Middlesex, HA6 2RN, UK.

Email: peterhoskin@nhs.net 